\title{
Evaluación de la calidad de suelo en sistemas de cultivo-arboles-pastos, micro cuenca Tecomapa, Somotillo-Nicaragua
}

\section{Soil quality assessment in culture-tree-pasture systems, micro Cuenca Tecomapa, Somotillo-Nicaragua}

\author{
Esteban Antonio Avilés Silva ${ }^{1}$, Reynaldo Bismarck Mendoza Corrales ${ }^{1}$, Cesar Aguirre ${ }^{1}$, Rein Van der Hoek ${ }^{2}$, Martin \\ Mena $^{2}$, Orlando Téllez Obregón ${ }^{2}$
}

IIng. ORCID: https://orcid.org/0000-0003-3936-6811 / 1 ORCID: https://orcid.org/0000-0003-4382-7983 / 1 ORCID: https://orcid.org/00000002-9363-6627 (Universidad Nacional Agraria) 2 ORCID: https://orcid.org/0000-0003-4528-7669 (Centro Internacional de Agricultura Tropical).

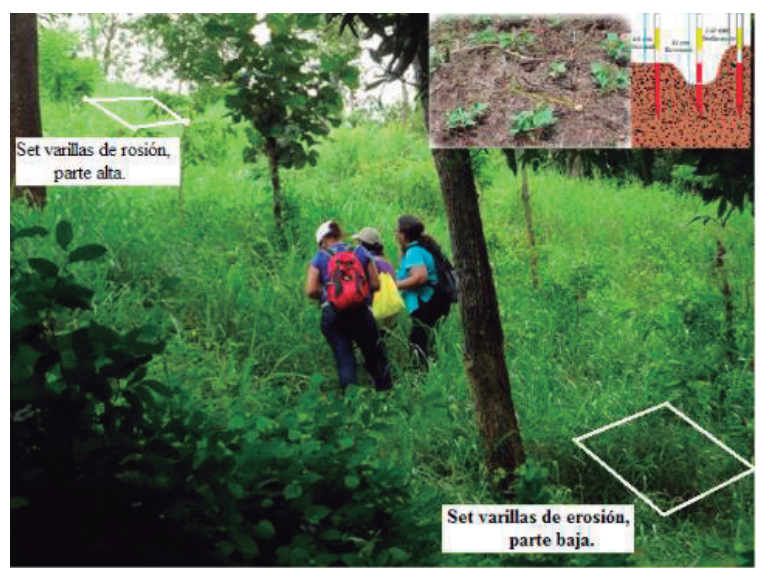

\section{RESUMEN}

Con el objetivo de evaluar los efectos de los sistemas de cultivo-arboles-pastos sobre el rendimiento de maíz y frijol, y la calidad de suelo, en la micro cuenca Tecomapa, fueron establecidas cinco parcelas experimentales: quema tradicional $(\mathrm{Q})$; sistema agroforestal Quesungual (SAQ); bosque secundario (Bs); potreros mejorados con Brachiaria sp - Marandú, (Pm); y sistema silvopastoril (SSP). En las cuales, dos sub parcelas localizadas en la parte alta y baja del relieve fueron establecidas para evaluar calidad de suelo y la erosión laminar, en 2011, 2012 y 2013. Como resultado, la tasa de erosión fuera de la parcela, únicamente ocurrió en el sistema de quema (Q) con $28.3 \mathrm{t} \mathrm{ha}^{-1} \mathrm{a}^{-1}$. La tasa de erosión dentro de la parcela fue moderada 31.98 y $42.97 \mathrm{t} \mathrm{ha}^{-1} \mathrm{a}^{-1}$, en los sistemas BS y Pm, y alta 62.97 y $121.79 \mathrm{t} \mathrm{ha}^{-1} \mathrm{a}^{-1}$, en los sistemas SAQ y SSP. La calidad de los suelos fue influenciada por los contenidos de materia orgánica en el suelo (MOS) mayores a $4000 \mathrm{~kg} \mathrm{ha}^{-1}$ en los sistemas SAQ, SSP y Bs. Este indicador fue correlacionado negativamente con el incremento de arcilla en el horizonte superficial de los suelos con pastura. Finalmente, los indicadores de calidad de suelo asociados al rendimiento del cultivo de maíz y frijol fueron el espesor orgánico de suelo, las tasas de infiltraciones rápidas, y la erosión de suelos.

Palabras claves: bosque secundario, sistemas agroforestales, manejo de cultivos, erosión laminar, indicadores de calidad de suelo.
In order to evaluate the effects of cropping systems-tree-grass on soil quality and soil erosion on three farms in the micro basin Tecomapa; were established five experimental plot: traditional burning (Q); Quesungual (SAQ) agroforestry system secondary forest (Bs); improved pastures with Brachiaria sp-Marandu (Pm); and silvopastoral system (SSP). In which two sub plots located in the high and lower position was monitoring soil quality and soil erosion sheet during 2011, 2012 and 2013. As a result, the rate of soil erosion out site was moderately only in the system of agricultural burning (Q) with $28.3 \mathrm{tha}^{-1} \mathrm{a}^{-1}$. The in soil erosion rate in site was moderate between 10-50 $\mathrm{t} \mathrm{ha}^{-1} \mathrm{a}^{-1}$, in the BS and Pm systems, and high (50 to $200 \mathrm{t} \mathrm{ha}^{-1} \mathrm{a}^{-1}$ ) in the SAQ and SSP systems. The soil quality was influenced by the contents of SOM, contents that were greater than $4000 \mathrm{~kg} \mathrm{ha}^{-1}$ in the SAQ, SSP and B systems. This indicator was negatively correlated with increasing clay in the surface horizon of soils used pasture by previous erosion. Finally, soil quality indicators that increase crop yields of corn and beans were the high the organic thickness of soil, rapid soil infiltration rates, and the soil erosion rate.

Key words: Secondary forest, agroforestry system, crop management, sheet soil erosion, indicators of soil quality indicator.
Recibido: 25 de mayo del 2018

Aceptado: 17 de septiembre del 2018

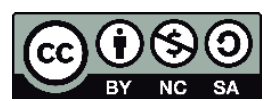

Los artículos de la revista La Calera de la Universidad Nacional Agraria, Nicaragua, se comparten bajo términos de la licencia Creative Commons: Reconocimiento, No Comercial, Compartir Igual. Las autorizaciones adicionales a las aquí delimitadas se pueden obtener en el correo freddy.aleman@ci.una.edu.ni

C Copyright 2018. Universidad Nacional Agraria 


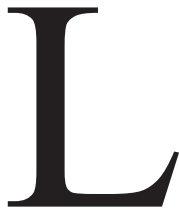

as tierras de laderas en Nicaragua, por ser densamente pobladas, ejercen mayor presión sobre los recursos naturales, por suministro de alimentos, bienes y servicios. En el municipio de Somotillo, la seguridad alimentaria se basa en la producción de maíz, fríjol, leche y carne. Producción que según la percepción local es afectada por la baja fertilidad del suelo y la erosión inducida por la escorrentía superficial. Como estrategia de manejo sostenible de suelos en el año 2000, fueron introducidos en la zona sistemas agroforestales para granos básicos y pasturas. Sin embargo, sus efectos ambientales y productivos eran desconocidos. Es así que en 2010 las entidades CIAT, UNA e INTA en alianza, diseñaron una investigación en campo, con el propósito de evaluar el efecto de los sistemas agroforestales sobre el rendimiento de maíz, frijol y pasturas. Esta investigación incluyó, evaluar la dinámica de erosión y la calidad de suelo generada por los sistemas agroforestales versus los sistemas tradicionales y el bosque secundario. Dichos objetivos son documentados en el presente estudio.

\section{MATERIALES Y MÉTODOS}

Características del sitio de estudio. El estudio fue realizado de abril 2011 a noviembre del 2013, en tres fincas de la microcuenca Tecomapa municipio de Somotillo, Chinandega. Localizada entre las coordenadas $13^{\circ} 8^{\prime} 25^{\prime}$ a $13^{\circ} 10^{\prime} 03^{\prime \prime}$ de latitud norte y $86^{\circ} 51^{\prime} 35^{\prime \prime}$ a $86^{\circ} 51^{\prime} 35^{\prime \prime}$ de longitud oeste, con un área de $14.04 \mathrm{~km}^{2}$ (INTA, 2005).

De clima de bosque seco tropical, (Holdrige, 1987), con precipitación promedio anual entre 1400 a 1600 $\mathrm{mm}$. Suelos de origen volcánico, arcillosos friables, clasificados como; Alfisoles (36.24\%), Molisoles (16.2\%), Vertisoles (0.38\%), asociación Entisoles-Molisoles (15.62 \%), asociación Alfisol-Entisol (35.85\%), y la asociación Molisol-Alfisol (26.27\%) del área en estudio. (Rodriguez et al., 2010). Topografía irregular, con pendientes entre 50 y $80 \%$ y elevaciones entre los 200 y $500 \mathrm{~m}$. La cobertura de uso es $30 \%$ agrícola, $40 \%$ pasturas tradicionales, y $30 \%$ bosque.

Las parcelas se establecieron en las fincas de: Roberto Pineda, Isidro Zúñiga y Teresa Rodríguez, con los siguientes usos y manejos: parcelas de quema (Q), donde se aplica tala y quema para cultivar maíz y frijol fertilizado, sistema Quesungual (SAQ) cultivada con maíz y frijol con árboles de la zona, ambas con área de 0.11 hectáreas. Sistema bosque secundario (Bs) en 0.5 ha, sistema con pasturas tradicionales y silvo pastoriles en 0.70 ha, con pasto mejorada (Brachiaria $s p$ ).

Monitoreo de calidad de suelo y erosión. En cada parcela se establecieron dos set de 10 varillas localizadas en la parte alta y baja, para un total de 100 varillas por finca, a una distancia mínima promedio de $15 \mathrm{~m}$ entre cada set aguas abajo. Método originado en el Colegio Postgraduados Chapingo, 1982, y modificado a diferentes topografías de Nicaragua por (Mendoza y Tinoco, 1994).

La erosión laminar se midió anualmente, y se recolectaron muestras de suelos alteradas (en bolsas) a una profundidad de $10 \mathrm{~cm}$, para evaluar contenidos de materia orgánica del suelo (MOS, método Wallkley and Black), densidad aparente y textura del suelo (método gravimétrico y Bouyuco). A la vez se midieron los indicadores: infiltración del agua en el suelo ( $\mathrm{min} / \mathrm{pul})$, pendiente (\%), espesor orgánico del suelo $(\mathrm{cm})$ en la parte alta y baja de cada parcela, utilizando métodos publicados en la guía de calidad de suelo por Mendoza (2014). Finalmente se midió el rendimiento de maíz y frijol en un área útil $\left(70 \mathrm{~m}^{2}\right)$.

La lámina de erosión total es la diferencia entre las lecturas de la lámina de suelo acumulada y la lámina erosionada, y se aplica la siguiente ecuación: $E v=D a^{*} H e^{*} A$.

Donde:

$\mathrm{Ev}=$ erosión laminar volumétrica $\left(\mathrm{t} \mathrm{ha}^{-1}\right), \mathrm{Da}=$ densidad aparente $\left(\mathrm{g} \mathrm{cm}^{-3}\right), \mathrm{He}=$ altura de suelo erosionado o depositado $(\mathrm{cm}), \mathrm{A}=$ área de medida $\left(\mathrm{m}^{2}\right)$.

Análisis de datos. Se analizaron 300 datos anuales de erosión laminar, convertidos a valores volumétricos ( $\mathrm{t} \mathrm{ha}^{-1}$ año-1), y 90 datos anuales para evaluar los indicadores de calidad de suelo: pendiente (\%), infiltración de agua en el suelo (min pul-1), espesor orgánico $(\mathrm{cm})$, contenido de arcilla (\%), acidez o $\mathrm{pH}$, y contenido de $\operatorname{MOS}\left(\mathrm{kg} \mathrm{ha}^{-1}\right)$. El rendimiento de maíz (primera) y frijol (postrera) fue determinado anualmente con 12 datos, en los sistemas quema (Q) y Quesungual (SAQ). El modelo estadístico utilizado fue: $Y i j k=\mu i j k l+\beta i+\theta j+\alpha k+\tau l+E(i j k)$.

Donde:

$\mathrm{i}=3$ finca (posiciones en el relieve), $\mathrm{j}=5$ tratamientos (descritos previamente), $\mathrm{k}=2$ posición en las sub-parcelas (alta y baja), 1 = 3 años, Yijk = Es el valor medio de las observaciones medidas en los distintos Tratamientos de cada finca., $\mu \mathrm{ijk}=$ Efectos de las medidas muestral sobre el modelo, $\beta \mathrm{i}=\mathrm{El}$ efecto de la i-ésimo (finca), $\theta \mathrm{j}=\mathrm{El}$ efecto de la j-ésimo (tratamientos), $\alpha \mathrm{k}=\mathrm{El}$ efecto del $\mathrm{k}$-ésimo (Posición de las sub-parcelas), $\tau \mathrm{l}=\mathrm{El}$ efecto de los años, $\mathrm{y}$ $\varepsilon i j k=$ Es el error de $(\beta \theta \alpha)$ ijk.

Para explicar la relación entre indicadores de calidad de suelo, se utilizaron las técnicas de Correlación Múltiple y el Análisis de Componentes Principales. Los análisis fueron corridos en InfoStat, 2009, y graficados en 
SigmaPlot 12.0 versión libre. Los coeficientes de correlación se clasificaron de fuerte ( $>0.65$ a 1), moderada $(0.25$ a 0.64) y débil (0.0 a 0.24) según (Kiernan, 2010).

\section{RESULTADOS Y DISCUSIÓN}

Las pérdidas de suelos son más severas en las primeras etapas de los cultivos cuando el suelo está descubierto y expuesto al golpe directo de las gotas de lluvia (erosión hídrica), quien a su vez destruyen las propiedades físicas del suelo (Narro, 1994). El patrón de lluvia durante los años 2011, 2012 y 2013 fueron bimodales y similares a los reportados por (INETER, 2014); observándose mayor lluvia en los meses de septiembre a octubre 2012 (figura 1).

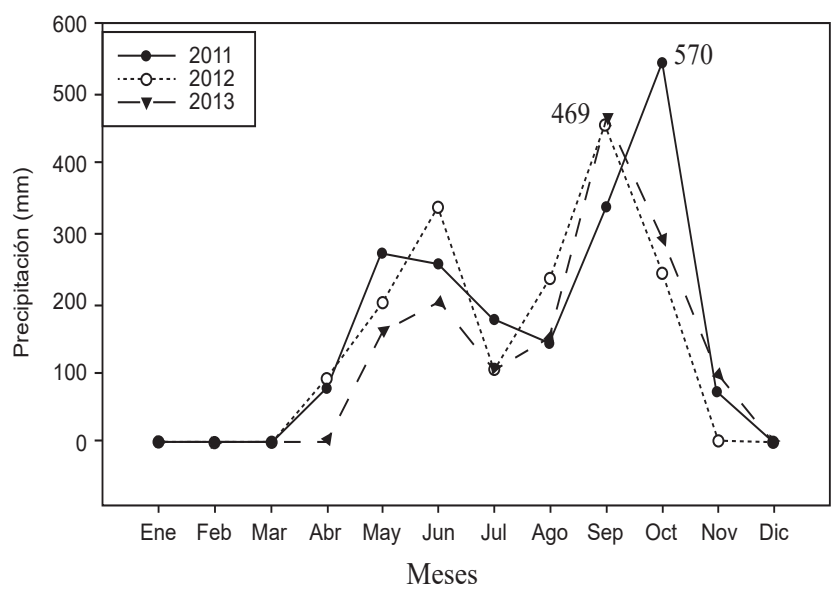

Figura 1. Registro de precipitación ( $\mathrm{mm})$, comunidades La Danta y La Flor, micro cuenca Tecomapa - Somotillo, 2011, 2012 y 2013.

Erosión de suelos. La tasa de erosión dentro de las parcelas fue significativamente diferente entre tratamientos $(p>\mathrm{F}=<0.0001)$, con balance total de 31.98, 121.79, $60.97 \mathrm{y}$ $42.11 \mathrm{t} \mathrm{ha}^{-1}$ año-1 $^{-1}$ para los sistemas de Bs, SAQ, SSP y Pm respectivamente (figura 2-A). Únicamente en el sistema de quema $(\mathrm{Q})$ ocurrió erosión de suelo fuera de la parcela con$28.30 \mathrm{t} \mathrm{ha}^{-1} \mathrm{a}^{-1}$. Tasas de erosión similares a las reportadas por Baltodano y Mendoza, en Lempira Sur-Honduras, en 2007 para sistemas con quema. La mayor tasa de erosión ocurrió en el año 2012 (figura 2-B; $p>\mathrm{F}=0.0012$ ). A nivel de finca la tasa de erosión dentro de las parcelas fue similar, con promedios entre 56.52 y $40.02 \mathrm{t} \mathrm{ha}^{-1} \mathrm{a}^{-1}$ (figura 2-C). El estudio revela que la dinámica de la erosión en el relieve es un 10\% mayor en la parte alta, si se compara con la parte baja, donde un $3 \%$ de ese material erosionado sale fuera de la parcela o sitio. Según, Afyuni et al., (1993), la dinámica de la erosión varía entre las posiciones altas y bajas dentro de un paisaje, siendo las posiciones altas de menor profundidad efectiva, debido a las mayores pérdidas de suelo. Esta tasa erosión dentro de las parcelas fue clasificada de moderada en los sistemas de Bs y Pm, y de alta en los sistemas SAQ y SSP. La tasa de erosión fuera de las parcelas fue clasificada de moderado únicamente en el sistema de quema agrícola, (FAO, 1980).
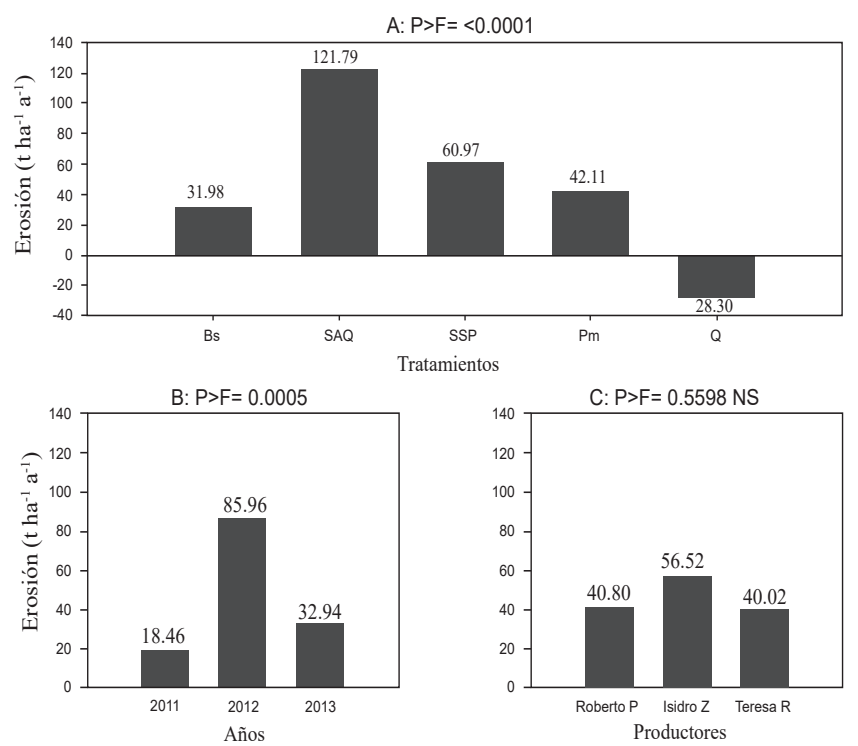

Figura 2. Tasa de erosión laminar en $\left(\mathrm{t} \mathrm{ha}^{-1} \mathrm{año}^{-1}\right)$ a nivel de sistema (A), anualmente (B) y por productores (C), micro cuenca Tecomapa, 2011, 2012 y 2013.

Indicadores de calidad de suelos. Un indicador de calidad de suelo, es una variable que resume o simplifica información relevante o condición de interés que cuantifica, mide y comunica, en forma comprensible la información (Doran y Parkin, 1996).

Los contenidos de MOS fueron similares entre tratamiento y significativamente diferentes entre fincas y años. Los valores promedios fueron: 48 090.2, 47 814.4, $46212.7,41345.3,38310.7 \mathrm{tha}^{-1}$ año $^{-1}$ en los tratamientos de Pm, Bs, SAQ, Q y SSP respectivamente (figura 3, A). Pavón (2008) reporta menores contenidos de MOS en la misma microcuenca en 2011. La tasa de infiltración fue reducida tres veces en las pasturas comparada con los bosques, probablemente por sobrepastoreo que compacta la superficie, erosionando el horizonte A, y aflorando el horizonte B, cargado con más arcilla. Por dichos fenómenos, la tasa de infiltración fue moderada en las sistemas con pasturas (SSP) y rápida en los bosques (21.94 contra $6.5 \mathrm{~min} \mathrm{pul}^{-1}$ de agua en los sistemas SSP y bosque respectivamente) (figura 4- A). 
A: $P>F=0.0007$

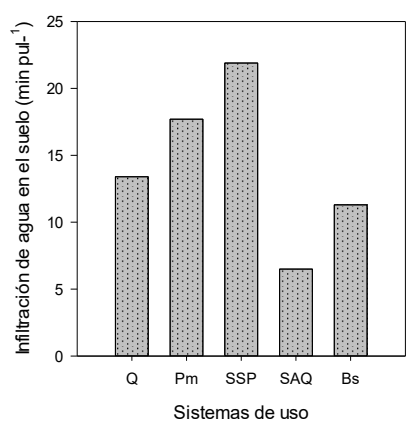

$B: P>F=0.0113$

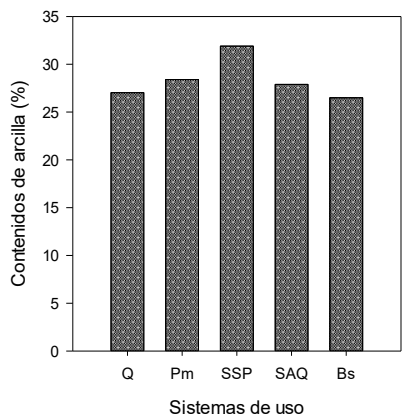

Figura 3. Indicadores de calidad de suelo relacionados a la fertilidad química de los suelos: (A) contenido de Materia orgánicas, (B) Espesor orgánico en el suelo. Monitoreo en la Micro cuenca Tecomapa 2012 y 2013.

\section{Análisis multivariados.}

Los sistemas de uso de la tierra en condiciones de laderas manejados inadecuadamente, conduce a un aumento acelerado de la erosión en el paisaje, inducido por el fenómeno de escorrentía superficial (FAO, 1994). El estudio identificó que la erosión de suelo afecta la calidad de los mismos, al ser removido el horizonte orgánico de la superficie y dar lugar al afloramiento del horizonte $\mathrm{B}$, cargado de más contenido de arcilla (cuadro 1). Este efecto produce una relación inversa y moderada entre los contenidos de materia orgánica en los suelos y arcilla ( $p>\mathrm{F}=0.0692 ; \mathrm{r} 2-0.85)$, y una velocidad de infiltración de agua que se reduce a menor espesor de suelo ( $p>\mathrm{F}=0.0290 ; \mathrm{r}=-0.68)$. Finalmente, se logró identificar que la erosión dentro de parcela reduce la acidez o $\mathrm{pH}(p>\mathrm{F}=$ $0.0049 ; \mathrm{r}=-0.81)$.

El análisis que reduce la dimensión y determina el grado de asociación entre los indicadores, es representado en el análisis de componente principal (APC). Para el mismo se tomó en cuenta una variabilidad de tres $\mathrm{CP}$ que suman $64.7 \%$ de la varianza, para explicar las interrelaciones. Según el análisis del CP1 en figura 5, los suelos usados con pasturas (Pm y SSP), presentan lenta infiltración asociada positivamente al alto contenido de arcilla en la superficie y a un leve incremento de la acidez, fenómeno probablemente asociado a compactación y erosión del horizonte superficial previamente. En el CP2, los suelos con mayor cobertura (Bs y SAQ), se caracterizan por poseer altos contenidos de MOS en la superficie, el cual disminuye al aumentar el gradiente de la pendiente y los contenidos de arcilla en la superficie. También se observa, que la práctica de quema (Q), se caracterizan por ser más susceptibles a la erosión laminar, perdiendo espesor orgánico y reduciendo levemente su acidez.

Relación indicadores de calidad de suelo y rendimiento de maíz y frijol. El rendimiento de los cultivos de maíz y frijol depende de la interacción de múltiples factores. En este estudio, el rendimiento de maíz y fríjol fueron ambos correlacionados negativamente a tasas lentas de infiltración de agua en el suelo $(p>\mathrm{F}=0.0630, \mathrm{r}=-0.94$, en maíz y r=-0.8 en frijol), confirmando que ambos cultivos prefieren suelos con buen drenaje o rápida velocidad de infiltración (cuadro 2). El rendimiento del maíz se ve favorecido en las partes bajas del relieve donde se depositan sedimentos $(p>\mathrm{F}=0.0563 ; \mathrm{r}=$ 0.94). Finalmente, el cultivo de fríjol se ve incrementado en áreas altas donde ocurre mayor erosión de suelo $(p>\mathrm{F}=0.0157 ; \quad \mathrm{r}=$ $0.98)$.

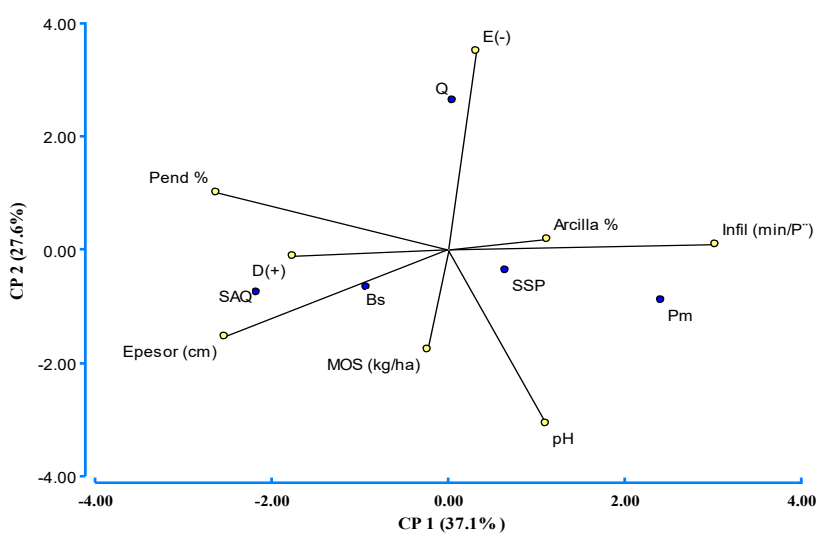

Figura 5. Análisis de componentes principales (CP1 y CP2), presentado en el primer y segundo plano, que relaciona los indicadores de calidad de suelo con los sistemas de uso del suelo. Análisis basado en 90 datos de muestreo. Tecomapa, 2011,2012 y 2013. 
Cuadro 1. Correlación múltiple entre sistemas de uso de la tierra y 8 indicadores de calidad de suelo, sobre la base de 90 datos. La fila en el numerador representa el coeficiente de correlación y por debajo la probabilidad. Microcuenca Tecomapa, 2011,2012 y 2013

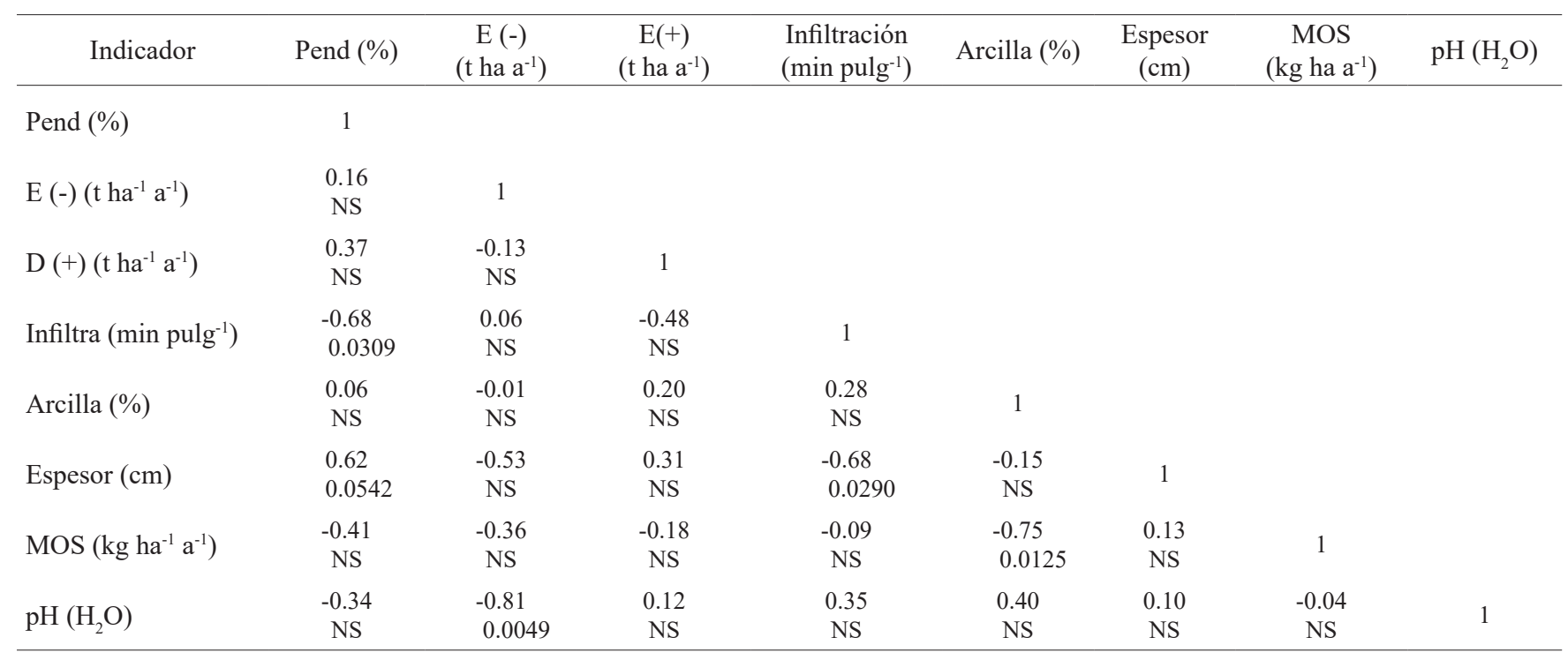

Cuadro 2. Correlación múltiple entre el rendimiento de maíz y frijol y 8 indicadores de calidad de suelo, sobre la base de 36 datos. La fila en el valor superior representa el coeficiente de correlación y el valor en la parte baja la probabilidad. Microcuenca Tecomapa, 2011, 2012 y 2013

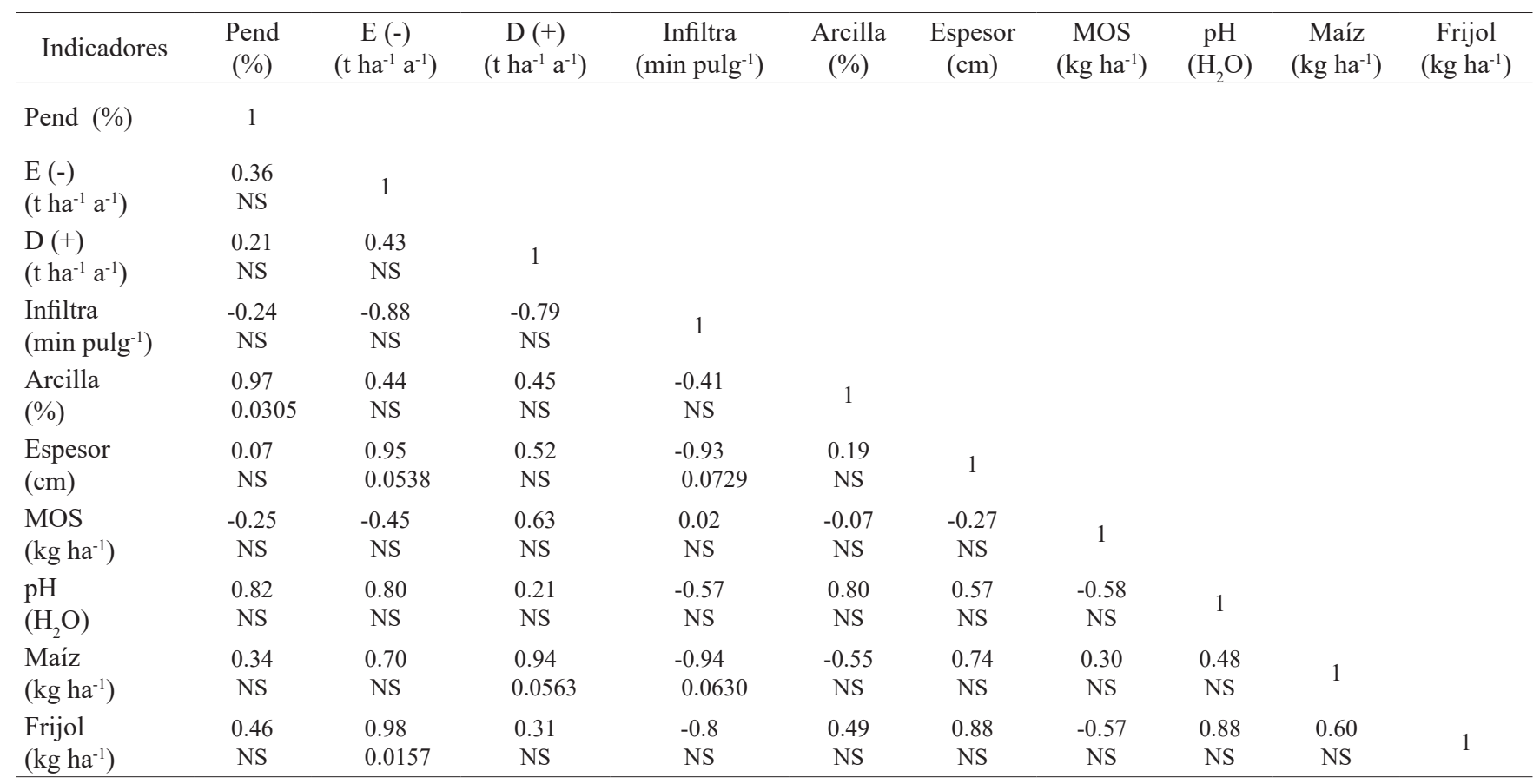




\section{CONCLUSIONES}

La erosión laminar medida en campos abiertos con el método de varillas mostro ser afectada por el tipo de cobertura vegetal, la pendiente del terreno y el uso de la tierra. Esto explica por que la erosión dentro de las parcelas fue mayor que la erosión que sale de las mismas, excepto en el sistema de quema agrícola por su poca cobertura y quema de la biomasa superficial.

La calidad de los suelos se observa afectadas en las pasturas por reducción de los contenidos de MOS, incremento de los contenidos de arcilla en la superficie y reducción de la velocidad de infiltración de agua en el suelo, lo cual sugiere aumentar la cobertura arbórea en áreas bajo pastos e identificar acciones para controlar el sobre pisoteo del ganado.

El rendimiento de maíz incrementa en áreas donde ocurre mayor depósito de sedimentos, o partes bajas del relieve, mientras que el frijol, se vio favorecido en áreas donde ocurre mayor erosión o partes altas del relieve. Sin embargo, ambos cultivos prefieren suelos con infiltraciones de agua rápida o bien drenada.

\section{REFERENCIAS BIBIOGRÁFICAS}

Afyuni, MM; Cassel, DK; Robarge, WP. 1993. Effect of landscape position on soil water and corn silage yield. Soil Sci. Soc. Amer. J. 57:1573-1580.

Baltodano, ME; Mendoza, RB. 2007. Evaluación y cuantificación de los efectos productivos y ambientales del sistema agroforestal Quesungual. (Inédito). CIAT, Managua, NI. 26 p.

Colegio de posgraduados, Chapingo, México. 1982. Manual de conservación de suelo y del agua. Segunda edición, Dirección de conservación del suelo yagua. Colegio de postgraduado, Chapingo, México. 1982. p 17.

Doran, JW; Parkin, TB. 1996. Quantitative indicators of soil Quality: a minimum data set p 25-37. In: Methods for assessing Soil Quality, SSSA Special Publication $\mathrm{N}^{\circ} 49$, Wisconsin, USA.

FAO (Organización de las Nacoines Unidas para Alimentación y la Agricultura). 1994. Land evaluation for forestry forestry paper. Rome: IT.

1980. Metodología provisional para la evaluación de la degradación de los suelos FAO-PNUMA-UNESCO. 86 p.

Forsythe, W. 1980. Física de suelos, manual dé laboratorio. Instituto Interamericano de Cooperación para la Agricultura. San José, CR.

Kiernan, D.H. 2010. Introductory Statistics for Environmental Sciencies. Lecture Supplement and Workbook. Kendall Hunt, publishing company. Dubuque, IA. 223p.

Holdrige, L. 1987. Ecología basada en zonas de vida. Tercera edición. Instituto interamericano de cooperación para la agricultura (IICA), San José, CR. 216 p.

INETER (Instituto Nicaragüense de Estudios Territoriales). 2014. Dirección General de Meteorología. Resumen Meteorológico anual. 1958 al 2014.

INTA. 2005. Diagnostico sub cuenca Tecomapa. Oficina de extensión Somotillo. Pacífico norte. Chinandega, Nicaragua. 121p.

Mendoza RB. 2014. Guía para el uso de indicadores de calidad de suelo. Universidad Nacional Agraria. Facultad de Recursos Naturales y del Ambiente. Managua, NI. 47 p.

Mendoza, ME; Tinoco, R. 1994. Evaluación de terraza de bordo y cultivos en curvas a nivel por el método clavos y rondanas. IRENA, SUWaR, PASOLAC. Managua, NI. 41 p.

Narro, E. 1994. Física de suelos con enfoque agrícola. Editorial México Trillas UAAAN 1994, 156 p.

Pavón T, JC. 2008. Aplicación de los principios del sistema agroforestal Quesungual en Nicaragua. Caracterización de la calidad física y química del suelo y susceptibilidad a la erosión en la Danta, Somotillo. Universidad Nacional de Colombia. $142 \mathrm{p}$

Rodríguez, II; Calero, A; Avilés, A; Mendoza, RB. 2008. Estudio detallado de suelos en Área de laderas por toposecuencia de la Microcuenca la Danta, Somotillo - Chinandega. (Inédito). Universidad Nacional Agraria. Managua, NI. 116 p. 